\title{
Accuracy of Force Exertion in Response to Demanded Forces Based on Subjective Information and Laterality
}

\author{
Takanori Noguchi $^{1, *}$, Shinichi Demura ${ }^{2}$, Masashi Omoya ${ }^{3}$ \\ ${ }^{1}$ Department of Industrial Business and Engineering, Fukui University of Technology, Fukui, Japan \\ ${ }^{2}$ Graduate School of Natural Science \& Technology, Kanazawa University, Kanazawa, Japan \\ ${ }^{3}$ Guraduate School of Human \& Socio-Environmental Studied, Kanazawa University, Kanazawa, Japan \\ *Corresponding author: t-noguchi@fukui-ut.ac.jp \\ Received October 08, 2014; Revised October 25, 2014; Accepted October 30, 2014
}

\begin{abstract}
The strength required for motor activities should be exerted effectively according to the type of activity and the load size. This study examined the relationship between the accuracy of handgrip exertion for demanded forces of $20 \%-80 \%$ of the maximal voluntary contraction (MVC) and laterality. Subjects were 100 healthy young males (mean age, $22.4 \pm 2.8$ years). After the handgrip MVC was measured, subjects attempted to exert a handgrip at demanded forces of 20,40,60, and $80 \%$ of the MVC. All tests were performed twice and with dominant and nondominant hands, and mean values were used for statistical analysis. Differences between demanded forces and exerted forces were converted into relative values based on each subject's MVC. Two-way repeated measures ANOVA showed significant interaction between demanded forces and laterality. In multiple comparison tests, smaller demanded forces were associated with larger errors only in the non-dominant hand. For demanded forces of $20 \%$ and $40 \%$ MVC errors were smaller for the dominant hand than for the non-dominant hand. The non-dominant hand is used less than the dominant hand in daily life and in sport activities. It is therefore not unexpected that a laterality-based difference in the accuracy of exerted force for each demanded force is found, and the accuracy of exerted force at low demanded forces was inferior for the non-dominant hand. In conclusion, there was a difference in the accuracy of exerted force for each demanded force for the non-dominant hand with a larger error at lower demanded forces. In particular, the accuracy of exerted force in response to demanded forces of $40 \%$ MVC or less was inferior in the non-dominant hand compared with that in the dominant hand. Laterality is therefore a significant factor in force response to lower demanded force values.
\end{abstract}

Keywords: laterality, handgrip, control the power

Cite This Article: Takanori Noguchi, Shinichi Demura, and Masashi Omoya, "Accuracy of Force Exertion in Response to Demanded Forces Based on Subjective Information and Laterality." American Journal of Sports Science and Medicine, vol. 2, no. 5 (2014): 190-193. doi: 10.12691/ajssm-2-5-3.

\section{Introduction}

Maximal muscle strength is rarely exerted during daily movements and activities, while we usually exert submaximal strength. However, in some activities including sports, it is often necessary to use moderate force as well as full power; for example, when striking a shuttlecock during a badminton game [1]. It has also been demonstrated that, if tennis players cannot adequately control the power exerted, the possibility of hitting the ball into the net or out of bounds is increased [2]. Therefore, it is important to understand the relationship between subjectively exerted force and actual output force, and the ability to exert force to a target value [2]. In the case of submaximal strength exertion, it is necessary to consciously or subconsciously estimate the degree of exertion required for a demanded task.

To perform subjective force exertion accurately, it is necessary to frequently experience the specific force output level [3]. The accuracy of subjective force exertion is based on past experiences and learning, and the ability to generate the appropriate output force is closely related to the ability to accurately control output force [4]. The accuracy of the output force generated increases through repeatedly receiving feedback on objective external information (measured values) and the comparison of information on internal sensory stimuli from muscles and tendons, joints, and the skin, with that external information.

However, in most activities of daily life, force exertion is subconsciously controlled and is not treated as the external information on force output values. Therefore, even when offered concrete values as targets for force output, it is not possible to accurately determine the difference (error) between the output values controlled by subjective information and the actual force output. Because the degree of the force exertion required to correct error is controlled in reference to maximal voluntary contraction (MVC) measured in advance, it is assumed that it would be more difficult to judge the force exertion level at lower demanded force values. 
Furthermore, controlled force exertion may be affected by laterality [5]. The concept of laterality means that one arm or leg is superior in strength and/or coordination $[6,7,8]$. Based on the preference for one limb over the other in activities of daily life, a dominant side develops $[9,10,11]$. Noguchi et al. [5] reported that the control of force exertion when adjusting the actual grip force to a wave shown on a computer display showed clearer laterality than maximal strength exertion.

The dominant hand is used more frequently than the non-dominant hand, and has greater and broader experiences of motor tasks performed in the past. Therefore, it is assumed that the accuracy of movement learning and output mechanisms involved in converting a perceived required force to an actual output force would be better in the dominant hand. However, in the experiment by Noguchi et al. [5], visual feedback information was given to the subjects. To date, the effect of laterality in controlled force exertion in response to subjective information has not been widely investigated. This study aimed to examine the accuracy of handgrip strength exertion at demanded forces of $20 \%-80 \%$ of the $\mathrm{MVC}$, and to assess the relationship with laterality.

\section{Methods}

\subsection{Subjects}

Subjects were 100 healthy young males (mean age, 22.4 \pm 2.8 years; mean height, $171.8 \pm 5.4 \mathrm{~cm}$; mean weight, $64.4 \pm 6.3 \mathrm{~kg}$; dominant handgrip strength, $51.1 \pm 8.3 \mathrm{~kg}$; and non-dominant handgrip strength, $47.7 \pm 8.3 \mathrm{~kg})$. All subjects were judged to be right-hand dominant based on Demura's Handedness Inquiry [12]. Before the measurements, the aim and procedures of the study were explained in detail and informed consent was obtained from all participants. This experimental protocol was approved by the Ethics Committee on Human Experimentation of the Faculty of Human Science, Kanazawa University (Ref. No. 2012-02).

\subsection{Procedures}

\subsubsection{Maximal Handgrip Strength Measurement}

A Smedley-type hand dynamometer (ED-D100R; Yagami Inc., Nagoya, Japan), which can measure strengths of 0-979 N (99.9 kg) with a $\pm 2 \%$ accuracy, was used to measure handgrip strength. The maximal handgrip strength test was carried out with the subject holding the arm straight down at the side of the body while standing and looking straight ahead, as described by Crosby et al. [13] and Nagasawa and Demura [8]. Subjects were instructed to exert maximal grip after a tester's signal. Handgrip strength was measured twice for each hand with a 2-min rest between trials. The maximum handgrip strength measured for each hand was used as the basis for the calculation of each demanded force.

\subsubsection{Subjective Exerted Force Measurement}

Demanded forces selected for the subjective exertion force test were $20 \%, 40 \%, 60 \%$, and $80 \%$ of the MVC. Two trails separated by a 2-min rest were performed for each demanded force. Measurements were performed with increasing demanded forces, starting with 20\% MVC. The measurements were performed with the dominant hand and the non-dominant hand for all subjects. The measurement device was covered, and so the subjects were not provided with feedback on the measurement values.

\subsection{Evaluation Variable}

The maximal handgrip strength differs among individuals. Therefore, we used the relative error (\%) as the evaluation variable. It was calculated using the following formula:

the relative error $(\%)=[\{($ subjective exerted force

demanded value) / maximal handgrip strength $\} \times 100$ ]

\subsection{Statistical Analysis}

Intraclass correlation coefficients (ICC) for each test were calculated to evaluate trial-to-trial reliability. Twoway repeated measures analysis of variance (ANOVA: demanded force $\times$ dominant/non-dominant hand) was used to reveal the differences among means for each condition. When a significant interaction or main effect was found, multiple comparison tests were performed using Tukey's honestly significant difference method. In addition, regression and intercept coefficients were calculated using the mean errors for each condition. Statistical significance $(\alpha)$ was set at $\mathrm{p}<.05$.

\section{Results}

Table 1 shows the ICCs for each demanded force. All variables showed high ICCs $>0.71$ (ICC $=0.71-0.91$ ).

Table 1. ICCs of each demand value

\begin{tabular}{|c|c|c|c|c|c|c|c|c|c|c|c|c|}
\hline & \multicolumn{3}{|c|}{$20 \% \mathrm{MVC}$} & \multicolumn{3}{|c|}{$40 \% \mathrm{MVC}$} & \multicolumn{3}{|c|}{$60 \%$ MVC } & \multicolumn{3}{|c|}{$80 \%$ MVC } \\
\hline & ICC & $\mathrm{F}$ & value $\mathrm{p}$ & ICC & $\mathrm{F}$ & value $\mathrm{p}$ & ICC & $\mathrm{F}$ & value $\mathrm{p}$ & ICC & $\mathrm{F}$ & value $\mathrm{p}$ \\
\hline Dominant hand & 0.84 & 2.50 & 0.12 & 0.73 & 3.29 & 0.07 & 0.75 & 1.93 & 0.17 & 0.71 & 2.38 & 0.13 \\
\hline non-dominand hand & 0.91 & 3.20 & 0.08 & 0.73 & 0.02 & 0.90 & 0.73 & 2.33 & 0.13 & 0.73 & 1.04 & 0.31 \\
\hline
\end{tabular}

Table 2. The results of two-way ANOVA (demanded value $x$ dominant/non-dominant hand)

\begin{tabular}{|c|c|c|c|c|c|c|c|c|}
\hline & $20 \%$ MVC & $40 \%$ MVC & $60 \%$ MVC & $80 \%$ MVC & \multicolumn{4}{|c|}{} \\
\hline Unit (\%) & Mean SD & Mean SD & fvtean SD & K/tean SD & & F & P & post-hoc \\
\hline do minant hand & 8.76 .7 & 7.24 .9 & 7.65 .8 & 7.65 .8 & F1 & 15.99 " & 0.01 & non-do minant: $20>40>60>80 \%$ MVC \\
\hline non-dominant hand & 13.68 .2 & 10.46 .7 & 9.15 .2 & 9.15 .2 & F2 & 34.75 " & 0.01 & $20,40 \%$ MVC: $\pm \mathrm{i}$ minant < no n- $\pm \mathrm{i}$ minant \\
\hline & & & & & IN & 14.31 " & 0.01 & \\
\hline
\end{tabular}

F1=demanded value, F2=dominant/non-dominant hand, IN=interaction 
Table 2 shows the results of two-way ANOVA and multiple comparison tests. A significant interaction was found $(F(3,297)=14.3, p<0.05)$. Multiple-comparison tests showed that, in the non-dominant hand, the error for a demanded force of $20 \%$ MVC was larger than that for all other demanded forces, and the error at 40\% MVC was larger than at $80 \% \mathrm{MVC}$. The errors for the non-dominant hand for demanded forces of 20\% MVC and 40\% MVC were larger than that for the dominant hand. Regression analysis of the errors for each demanded force in the nondominant hand was significant (regression: -2.14 , $\mathrm{p}<0.05$, intercept: 15.35, $\mathrm{p}<0.05$ ).

\section{Discussion}

This study aimed to examine the accuracy of handgrip strength exertion to demanded forces of $20 \%-80 \%$ of the MVC and the effect of laterality. Maximal force exertion is not required for the fine control of movements. When performing activities of daily life, the output force required is generally subconsciously estimated and exerted. In the present study, the force required was controlled in reference to the MVC measured previously. Therefore, it was expected that it would be more difficult to judge the force exertion level at lower demanded forces. In addition, because the dominant hand is used more frequently in daily life, with greater accumulated experiences of motor tasks performed in the past, the accuracy of force exertion would be expected to be higher than in the non-dominant hand. We hypothesized that the higher the demanded force, the lower the error between the demanded and actual force, and that the accuracy of applying the demanded force would be inferior in the non-dominant hand compared with that in the dominant hand.

In human motor learning, there are two types of information: that based on internal sensory stimuli including information from muscles and tendons, joints and the skin, and that based on external stimuli including visual information. Providing feedback based on information from internal or external stimuli, or both repeatedly, improves the accuracy of movements [14]. For example, in the case of voluntary force exertion, when exerting, the MVC subjects are conscious of internal information and have subjective control over the exerting force [2]. However, when there is no external information, it is assumed that a small force exertion will incur a greater error because the force required is considerably less than the MVC. This hypothesis was accepted in the non-dominant hand, but not in the dominant hand. In the non-dominant hand, the minimum demanded force of $20 \%$ MVC was associated with the largest error, and as the demanded force increased, the error decreased. However, for the dominant hand, there were no significant differences among the errors for each demanded value. Laterality was demonstrated for demanded forces below 40\% MVC.

It has been suggested that the development of controlled force exertion ability is affected by acquired factors and that laterality becomes more marked over time [15]. Because the dominant hand is more frequently used in daily life and is more exposed to various stimulations, it was predicted that the dominant hand would perform better in matching demanded forces owing to their experiences. The non-dominant hand tends to exert excess force with more muscle rigidity than the dominant hand. Furthermore, because the non-dominant hand does not develop motion output mechanisms from the central nervous system to the effector to the extent of the dominant hand, it is difficult to control force exertion with the non-dominant hand.

The above mechanisms can explain the differences between the dominant and non-dominant hands. However, all subjects in the present study were right-hand dominant. If subjects were left-handed dominant or ambidextrous, different results may have been obtained. Therefore, in the future, a reexamination of the present problem using subjects with a full range of laterality is required to generalize of the experiment's result.

This study demonstrated that, when attempting to match output forces to demanded forces, regardless of the size of demanded forces, errors in the dominant hand were almost constant. In contrast, only the non-dominant hand errors differed among the demanded forces, with a larger error for lower demanded forces. Compared with the dominant hand, the non-dominant hand showed inferior accuracy for matching exerted force to demanded force at values of $40 \%$ MVC or less; thus, it showed that laterality is observed.

\section{Acknowledgement}

The authors would like to thank Enago (www.enago.jp) for the English language review.

\section{References}

[1] Kaneko, M., Furukawa, S., Ito, K. and Muraki, Y., “Adjustment of the strength to the stroke movement in the badminton: a difference of every school year of the female junior high school badminton player,” The Japan Journal of Sport Methodology, 21 (2). 157-165. 2008.

[2] Ohtsuki, T., "Skillful of Brain and Voluntary Exercise," 46 (6). 444-446. 1996.

[3] Seki,T. and Ohtsuki,T., "Reproducibility of subjectively graded voluntary isometric muscle strength in unilateral and simultaneous bilateral exertion,” Ergonomics, 38 (9). 1867-1876. Sept 1995.

[4] Sadamoto, T. and Ohtsuki, T., "Accuracy of Output Control in Jumping: Characteristics in Grading and Reproduction of Distance,” Japan Journal of Physical Education, 22 (4). 215-229. 1977.

[5] Noguchi, T., Demura, S. and Aoki, H., "Superiority of the dominant and nondominant hands in static strength and controlled force exertion,” Perceptual and Motor Skills, 109 (2). 339-346. Oct 2009.

[6] Chi J.G., Dooling E.C. and Gilles F.H., "Left-right asymmetry of the temporal speech areas of human fetus," Archives of Neurology, 34 (6). 346-348. Jun 1977.

[7] Demura, S., Yamaji, S., Goshi, F. and Nagasawa, Y., "Lateral dominance of legs in maximal muscle power, muscular endurance, and grading ability” Perceptual and Motor Skills, 93 (1). 11-23. Aug 2001.

[8] Nagasawa, Y. and Demura, S., "Development of an apparatus to estimate coordinated exertion of force" Perceptual and Motor Skills, 94. 899-913. Jun 2002.

[9] Oldfield, R.C., "The assessment and analysis of handedness: The Edinburgh Inventory,” Neuropsychologia, 9 (1). 97-113. Mar 1971.

[10] Annett M., "A classification of hand preference by association analysis," The British Journal of Psychology, 61 (9). 303-321. Aug 1970.

[11] Chapman, L.J. and Chapman, J.P., "The measurment of handedness" Brain and Cognition, 6 (2). 175-183. Apr 1987. 
[12] Demura, S., Sato, S. and Nagasawa,Y., "Re-examination of useful items for determining hand dominance," Medica Italiana Archivio per le Scienze Mediche, 169-177.

[13] Crosby, C.A., Wehbe, M.A. and Mawr, B., "Hand strength: normative values" The Journal of hand surgery, 19 (4). 665-670. Jul 1994.
[14] Richard, A., Schmidt, Craig, A. Wrisberg, Motor Learning and Performance With Web Study Guide - 4th Edition: A SituationBased Learning Approach, Human Kinetics; 4 edition, 2007

[15] Ohtsuki, H., Hasebe, S., Okano, M. and Furuse, T., "Comparison of surgical results of responders and non-responders to the prism adaptation test in intermittent exotropia," Acta Ophthalmology Scandinavica, 75 (5). 528-531. Oct 1997. 